\title{
Implications of a high-mass diphoton resonance for heavy quark searches
}

\author{
Shankha Banerjee, Daniele Barducci, Geneviève Bélanger and Cédric Delaunay \\ LAPTh, Université Savoie Mont Blanc, CNRS B.P. 110, \\ F-74941 Annecy-le-Vieux, France \\ E-mail: banerjee@lapth.cnrs.fr, daniele.barducci@sissa.it, \\ belanger@lapth.cnrs.fr, delaunay@lapth.cnrs.fr
}

\begin{abstract}
Heavy vector-like quarks coupled to a scalar $S$ will induce a coupling of this scalar to gluons and possibly (if electrically charged) photons. The decay of the heavy quark into $S q$, with $q$ being a Standard Model quark, provides, if kinematically allowed, new channels for heavy quark searches. Inspired by naturalness considerations, we consider the case of a vector-like partner of the top quark. For illustration, we show that a singlet partner can be searched for at the $13 \mathrm{TeV}$ LHC through its decay into a scalar resonance in the $2 \gamma+\ell+X$ final states, especially if the diphoton branching ratio of the scalar $S$ is further enhanced by the contribution of non coloured particles. We then show that conventional heavy quark searches are also sensitive to this new decay mode, when $S$ decays hadronically, by slightly tightening the current selection cuts. Finally, we comment about the possibility of disentangling, by scrutinising appropriate kinematic distributions, heavy quark decays to $S t$ from other standard decay modes.
\end{abstract}

KeYwords: Beyond Standard Model, Heavy Quark Physics

ARXIV EPRINT: 1606.09013 


\section{Contents}

1 Introduction 1

2 The model 2

3 A new channel for VLQ searches 4

4 Impact on other VLQ searches $\quad 8$

$\begin{array}{lll}5 & \text { Conclusions } & 10\end{array}$

\section{Introduction}

Vector-like quarks (VLQ) are predicted in several extensions of the standard model (SM), from extra dimensions models to composite Higgs models and including non-minimal SUSY extensions or grand unified theories [1-10]. Moreover, many of these models feature an extended scalar sector to which new fermions, in particular VLQs, can couple. A singlet in association with VLQ has also been shown to help stabilize the electroweak vacuum $[11,12]$. This has two important phenomenological consequences. First, charged VLQs will induce one-loop couplings of scalars to two photons or two gluons. Thus, the scalar $S$ produced via gluon-fusion will have a distinctive signature at the LHC in the diphoton channel. Early hints of an excess in this channel for a resonance at $750 \mathrm{GeV}$ in the first $13 \mathrm{TeV}$ energy run at the Large Hadron Collider (LHC), reinforced by the reanalysis of the $8 \mathrm{TeV}$ Run [13-18], have motivated a host of dedicated studies both for the cases of the new resonance being a spin zero or two [10, 19-32]. Uncoloured states were also considered as they can also lead to a large enough diphoton cross section by only increasing the diphoton partial width [22], albeit with $S$ couplings dangerously approaching the non-perturbative regime $[33,34]$. These hints have however not been confirmed, thus leading to an upper bound for the cross section for the production of a new scalar of $\sigma_{p p \rightarrow S \rightarrow \gamma \gamma}<1.5-3 \mathrm{fb}$ for a narrow resonance with a mass of $750 \mathrm{GeV}$ [35, 36]. Second, it opens up the possibility for VLQs to decay into a scalar and a quark. Moreover, this VLQ decay will lead to a very clean signature if the scalar has a significant diphoton branching ratio (BR). Typically BRs of the order $10^{-3}-10^{-2}$ are required, which are roughly in the same ball park as the diphoton BR of the SM Higgs. We entertain in this letter a generic scenario where a single VLQ is coupled to a new scalar which has a non-negligible decay into diphotons. For definiteness and motivated by early hints we will consider the case where the scalar has a mass of $750 \mathrm{GeV}$. Although similar analysis could be done for spin-2, for simplicity we consider only a scalar resonance.

Heavy VLQs are mainly pair produced via QCD interaction at the LHC, with a cross section that depends only on the VLQ mass. Decays of the VLQs are typically governed 
by mixings with SM quarks, yielding final states with SM quarks and electroweak (EW) gauge or Higgs bosons. $8 \mathrm{TeV}$ LHC searches of VLQs with charge $2 / 3$ and $-1 / 3$ and mixing exclusively with third generation quarks excludes VLQ masses below $700-950 \mathrm{GeV}$, depending on the VLQ branching ratio (BR) configuration [37-39], while limits on pairproduced VLQs mixing with the light quark generations, in the $400-700 \mathrm{GeV}$ range, are much weaker [40]. ${ }^{1}$ The sensitivity to high mass VLQs within the early $13 \mathrm{TeV}$ data is still slightly below, yet quickly reaching, that of the $8 \mathrm{TeV}$ run [42]. As we argue below VLQ decays into $S$ and SM quarks, whenever accessible, typically dominate over EW channels. The existence of these channels would then affect the traditional search strategies for pair-produced VLQs [43-46] but, more importantly, their exploration would provide a complementary probe of the new physics sector associated with the new scalar (see e.g. ref. [47]).

In this letter, we provide a first evaluation of the impact of a heavy diphoton resonance on VLQ searches. For definiteness in the numerical studies we will assume the mass of the scalar to be $750 \mathrm{GeV}$. Motivated by the SM hierarchy problem and naturalness of the EW scale, we focus here on the possibility that the new VLQ is mixing with third generation SM quarks. In particular, inspired by an approach exploited at LHC Run-1 for searching the VLQ $\rightarrow H t$ decay [37], we study the sensitivity of the LHC Run-2 to the process ${ }^{2}$ $p p \rightarrow \mathrm{VLQ} \overline{\mathrm{VLQ}} \rightarrow S t+Y$ ( $Y$ denoting any possible VLQ decay product) in the $2 \gamma+\ell+X$ final states. Finally, we study the implications of the possibly large VLQ $\rightarrow$ St decay rate on other VLQ searches in conventional channels. We show that existing analyses could be sensitive to this decay mode, albeit with tighter kinematical selections cuts.

\section{The model}

We consider a simplified model that contains, in addition to the SM fields, a neutral scalar singlet $S$, and a VLQ $T, T^{c}$ transforming as $(\mathbf{3}, \mathbf{1}, 2 / 3)$ under $\mathrm{SU}(3)_{c} \times \mathrm{SU}(2)_{L} \times \mathrm{U}(1)_{Y}$. The model Lagrangian is $\mathcal{L}=\mathcal{L}_{\mathrm{SM}}+\mathcal{L}_{S, T}$ where $\mathcal{L}_{\mathrm{SM}}$ is the SM Lagrangian and

$$
\begin{aligned}
\mathcal{L}_{S, T}= & \frac{1}{2}\left(\partial_{\mu} S\right)^{2}-\frac{m_{S}^{2}}{2} S^{2}+\bar{T}(i \not D-M) T \\
& -y_{S} S \bar{T}_{L} T_{R}-m \bar{t} T_{L}-y_{T}(\bar{q} \tilde{H}) T_{R}+\text { h.c. },
\end{aligned}
$$

where $H$ is the SM Higgs doublet, with $\tilde{H}=i \sigma_{2} H^{*}$, and $q$ and $t$ are the SM third generation quark doublet and top quark singlet, respectively. Both mixing parameters $m$ and $y_{T} v / \sqrt{2}$, with $v=\left(\sqrt{2} G_{\mathrm{F}}\right)^{-1 / 2} \approx 246 \mathrm{GeV}$, will trigger $T$ decays into SM states. Since the quark chirality of the decay products will not play any important role in our analysis, we thus consider for sake of simplicity a limit where only one mixing parameter is present and set $y_{T}=0$. In the large $M$ limit the physical top and heavy quark masses are approximately

$$
m_{t}^{2} \simeq \frac{y_{t}^{2} v^{2}}{2}\left(1-\frac{m^{2}}{M^{2}}\right), \quad m_{T}^{2} \simeq M^{2}\left(1+\frac{m^{2}}{M^{2}}\right),
$$

\footnotetext{
${ }^{1}$ See also [41] for a phenomenological study where bounds on VLQs mixing with light generations are obtained through the recast of supersymmetry inspired searches.

${ }^{2}$ Reference [48] has studied in details the implications of a diphoton resonance from the decay of a singly produced vector-like quark.
} 
where $y_{t}$ is the SM top Yukawa coupling, while the mixing angles for the left and right chirality components read approximately

$$
\begin{aligned}
& \tan 2 \theta_{R} \simeq \frac{2 m}{M}\left(1+\frac{m^{2}}{M^{2}}+\frac{y_{t}^{2} v^{2}}{2 M^{2}}\right), \\
& \frac{\tan 2 \theta_{L}}{\tan 2 \theta_{R}} \simeq \frac{y_{t} v}{\sqrt{2} M}\left(1-2 \frac{m^{2}}{M^{2}}\right) .
\end{aligned}
$$

Note that the two mixing angles are no longer related in the presence of a non-zero $\bar{q} \tilde{H} T_{R}$ operator.

In the model of eq. (2.1) the $S-g g$ and $S-\gamma \gamma$ interactions arise dominantly through a $T$ loop ${ }^{3}$ yielding the following amplitudes

$$
\mathcal{A}_{S \rightarrow g g}=\frac{\alpha_{s}}{3 \pi} c_{g} \delta^{a b}\left[\frac{m_{S}^{2}}{2}\left(\epsilon_{1} \cdot \epsilon_{2}\right)-\left(\epsilon_{1} \cdot p_{2}\right)\left(\epsilon_{2} \cdot p_{1}\right)\right],
$$

where $p_{1,2}^{\mu}$ and $\epsilon_{1,2}^{\mu}$ are the momenta and polarization vectors of the gluons, $a, b$ are colour indices and

$$
c_{g}=\frac{3 y_{S} c_{L} c_{R}}{4 m_{T}} F_{1 / 2}\left(\tau_{S}\right), \quad \tau_{S} \equiv \frac{m_{S}^{2}}{4 m_{T}^{2}} .
$$

A similar expression for $\mathcal{A}_{S \rightarrow \gamma \gamma}$ is obtained from eq. (2.4) through replacing $\alpha_{s} \rightarrow \alpha$, $\delta^{a b} \rightarrow 1$ and $c_{g} \rightarrow c_{\gamma}=6 Q_{T}^{2} c_{g}=8 c_{g} / 3$. The $F_{1 / 2}(\tau)$ form factor, with $F_{1 / 2}(0)=\frac{4}{3}$, is found e.g. in ref. [49]. The $S$ partial widths into $g g, \gamma \gamma$ and $t \bar{t}$ are thus

$$
\begin{aligned}
\Gamma_{S \rightarrow g g} & =\frac{\alpha_{s}^{2} m_{S}^{3}}{72 \pi^{3}}\left|c_{g}\right|^{2}, & \Gamma_{S \rightarrow \gamma \gamma}=\frac{\alpha^{2} m_{S}^{3}}{576 \pi^{3}}\left|c_{\gamma}\right|^{2}, \\
\Gamma_{S \rightarrow t \bar{t}} & =\frac{3 y_{S}^{2} s_{L}^{2} s_{R}^{2} m_{S}}{8 \pi} \beta_{t}^{3}, &
\end{aligned}
$$

where $\beta_{t} \equiv\left(1-\frac{4 m_{t}^{2}}{m_{S}^{2}}\right)^{1 / 2}$. Decays into $Z \gamma$ and $Z Z$ are also predicted with, up to $m_{Z} / m_{S}$ corrections, $\Gamma_{S \rightarrow Z \gamma} / \Gamma_{S \rightarrow \gamma \gamma} \simeq 2 \tan ^{2} \theta_{W}$ and $\Gamma_{S \rightarrow Z Z} / \Gamma_{S \rightarrow \gamma \gamma} \simeq \tan ^{4} \theta_{W}$, respectively, while $\Gamma_{S \rightarrow W W}=0 ; \theta_{W}$ is the weak mixing angle. Since $2 \tan ^{2} \theta_{W} \approx 0.6$ and $\tan ^{4} \theta_{W} \approx 0.1$ these decay modes will not significantly affect the $S$ total width, nor its diphoton BR since typically $\Gamma_{S \rightarrow \gamma \gamma} / \Gamma_{S \rightarrow g g} \simeq \mathcal{O}\left(\alpha^{2} / \alpha_{s}^{2}\right) \sim 0.005$.

The mixing operators in eq. (2.1) induces the decay of the heavy quark $T$ into $W b$, $Z t, h t$ and $S t$ final states, with rates

$$
\begin{aligned}
\Gamma_{T \rightarrow W b} & =\frac{\alpha s_{L}^{2} m_{T}}{16 \sin ^{2} \theta_{W}} \lambda_{b, W}^{1 / 2} \zeta_{b, W}, \\
\Gamma_{T \rightarrow Z t} & =\frac{\alpha s_{L}^{2} c_{L}^{2} m_{T}}{32 \sin ^{2} \theta_{W} \cos ^{2} \theta_{W}} \lambda_{t, Z}^{1 / 2} \zeta_{t, Z} \\
\Gamma_{T \rightarrow h t} & =\frac{y_{t}^{2} m_{T}}{64 \pi}\left(c_{R}^{2} s_{L}^{2}+c_{L}^{2} s_{R}^{2}\right) \lambda_{t, h}^{1 / 2} \xi_{t, h}, \\
\Gamma_{T \rightarrow S t} & =\frac{y_{S}^{2} m_{T}}{32 \pi}\left(c_{R}^{2} s_{L}^{2}+c_{L}^{2} s_{R}^{2}\right) \lambda_{t, S}^{1 / 2} \xi_{t, S}
\end{aligned}
$$

\footnotetext{
${ }^{3}$ There is also a top quark loop contribution which is suppressed by the small mixing angles $s_{L} s_{R}$ assumption (see later) and overall negligible.
} 
where $\lambda_{a, b} \equiv \lambda\left(m_{a}^{2} / m_{T}^{2}, m_{b}^{2} / m_{T}^{2}\right)$ with $\lambda(x, y)=1+x^{2}+y^{2}-2 x-2 y-2 x y$ and similarly for $\zeta_{a, b}$ and $\xi_{a, b}$ with $\zeta(x, y)=1+x^{2}-2 y^{2}+\frac{\left(1-x^{2}\right)^{2}}{y^{2}}$ and $\xi(x, y)=1+x^{2}-y^{2}+x s_{2 L} s_{2 R} /\left(c_{R}^{2} s_{L}^{2}+\right.$ $\left.c_{L}^{2} s_{R}^{2}\right)$. Note, in the limit $m_{T} \gg m_{t}, c_{L} \rightarrow 1$ and $\Gamma_{T \rightarrow Z t} \simeq \Gamma_{T \rightarrow H t} \simeq \frac{1}{2} \Gamma_{T \rightarrow W b}$ as expected from the equivalence theorem. In this limit, the value of $\operatorname{BR}(T \rightarrow S t)$ is thus essentially determined by $y_{S}^{2} / y_{t}^{2}$.

The simplified model of eq. (2.1) has three new parameters, which we choose to be $y_{S}$, the Yukawa coupling of the resonance with the VLQ, $m_{T}$ the physical VLQ mass and the RH mixing angle $\theta_{R}$. The loop-induced decay rates remain constant for $\theta_{R} \lesssim 0.1$, while for larger mixing values the partial width in $t \bar{t}$ becomes comparable with the $g g$ one, leading to an overly suppressed BR in $\gamma \gamma$. In order to ensure a large enough diphoton BR we fix $\theta_{R}=0.01$ for sake of definiteness. Neglecting the $S \rightarrow t \bar{t}$ contribution, the diphoton BR reads

$$
\mathrm{BR}(S \rightarrow \gamma \gamma) \simeq \frac{\Gamma_{S \rightarrow \gamma \gamma}}{\Gamma_{S \rightarrow g g}}=\frac{8 \alpha^{2}}{9 \alpha_{s}^{2}} \approx 4 \times 10^{-3}
$$

Note that the total $S$ width is dominated by the $g g$ channel and is quite small, typically sub-GeV.

The heavy quark BRs are almost independent of the mixing angles and, as already mentioned above, the BR into $T \rightarrow S t$ is essentially set by the ratio $y_{S}^{2} / y_{t}^{2}$. This mode starts to dominate for values of $y_{S} \sim 4(2)$ for $m_{Q}=1(1.5) \mathrm{TeV}$, as shown in figure 1. In the model under consideration $\operatorname{BR}(S \rightarrow \gamma \gamma)$ can attain a maximum rate of $\sim 0.4 \%$. It is however possible to increase the $\gamma \gamma$ decay rate, without modifying the heavy quark BRs by introducing extra states, for example vector-like leptons (VLL) that will only contribute to the $S \rightarrow \gamma \gamma$ amplitude. From the expression of the loop-induced diphoton partial width

$$
\Gamma_{S \rightarrow \gamma \gamma}=\frac{\alpha^{2} m_{S}^{3}}{576 \pi^{3}}\left|c_{\gamma}^{T}+c_{\gamma}^{L}\right|^{2},
$$

where $c_{g}^{T}$ is given in eq. (2.5) and $c_{\gamma}^{L}=\sum_{i} \frac{3}{2} y_{S}^{i} / m_{L}^{i} Q_{L^{i}}^{2} F_{1 / 2}\left(\tau_{S}^{i}\right)$ where the sum runs over all VLLs. Generalised to the case of heavy leptons with mass $m_{L}^{i}$, charge $Q_{L}^{i}$ and coupling $y_{L}^{i}$ to $\mathrm{S}$, we can derive the ratio of the partial widths including only the heavy quark, $\Gamma_{\gamma \gamma}^{T}$, to the one including in addition a heavy lepton, $\Gamma_{\gamma \gamma}^{T+L}$. For a unit charged VLL with the same coupling $y_{S}$ as the heavy quark and $m_{T}=1000 \mathrm{GeV}$, the ratio $\Gamma_{\gamma \gamma}^{T+L} / \Gamma_{\gamma \gamma}^{T}$ is $\sim 7$ for $m_{V L L}=500 \mathrm{GeV}$ and $\sim 3$ for $m_{V L L}=1000 \mathrm{GeV}$. In the following, we will thus treat the $S \rightarrow \gamma \gamma$ partial width (and hence the corresponding BR) as a free parameter that can be increased by up to an order of magnitude from the value obtained using only the $T$ quark.

\section{A new channel for VLQ searches}

The high $T \rightarrow S t$ BR attainable in this simplified scenario opens the possibility of searching for heavy quarks at the LHC through this unconventional decay channel, if a new scalar resonance $S$ to which the heavy quarks couple were to be discovered at the LHC. Here we study this possibility choosing for definiteness a specific value for the $S$ mass, namely $m_{S} \sim 750 \mathrm{GeV}$, and then commenting on how our proposed search strategy can be adapted for different $S$ mass assumptions. 


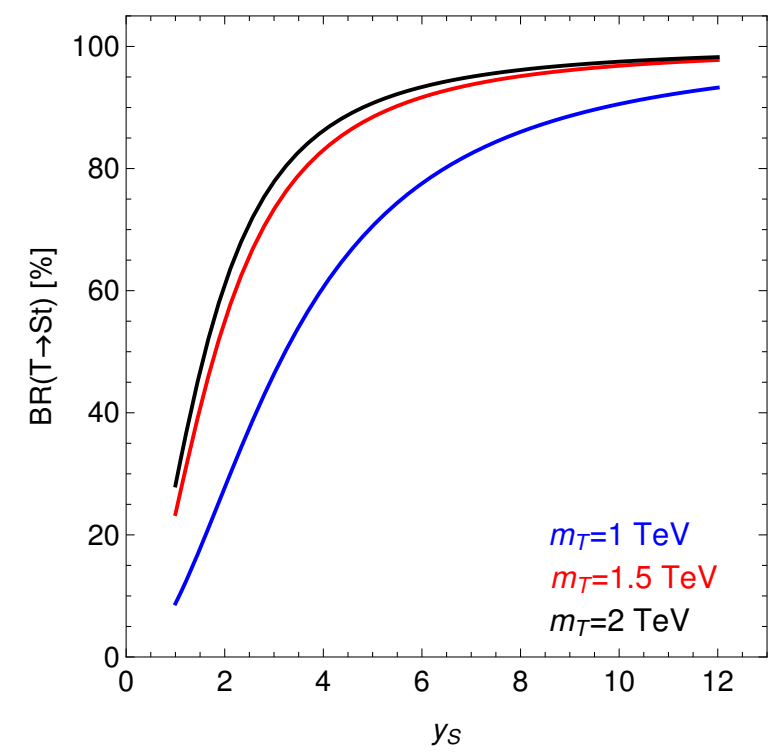

Figure 1. $\operatorname{BR}(T \rightarrow S t)$ as a function of the Yukawa coupling $y_{S}$ for $m_{T}=1 \mathrm{TeV}$ (blue), $1.5 \mathrm{TeV}$ (red) and $2 \mathrm{TeV}$ (black), assuming $\theta_{R}=0.01$.

During the $8 \mathrm{TeV}$ run of the LHC, the CMS collaboration performed a search for pair produced heavy quarks with charge $2 / 3$ decaying into all possible $W b, Z t$ and $H t$ combinations [37]. Among the various channel investigated, an analysis was optimised for events with one heavy quark decaying into $H t$, with the Higgs boson subsequently decaying into a $\gamma \gamma$ final state. Interestingly, despite the small $\operatorname{SM~} \operatorname{BR}(H \rightarrow \gamma \gamma) \sim 2 \times 10^{-3}$, this channel was exploited to set a limit of $\sim 600 \mathrm{GeV}$ on $m_{T}$, under the assumption that $\mathrm{BR}(T \rightarrow H t)=1$. We thus aim at exploring the sensitivity of the $13 \mathrm{TeV}$ LHC for a analogous search strategy based on the $T \rightarrow S t, S \rightarrow \gamma \gamma$ decay.

The main sources of background for the $8 \mathrm{TeV}$ CMS analysis are the resonant $t t H$ production, and the non resonant processes $\gamma \gamma t \bar{t}, \gamma \gamma t$ and $\gamma \gamma+$ jets. CMS enforces selection cuts that take advantage of the resonant production of the $\gamma \gamma$ pair in the signal topology, therefore substantially reducing the non resonant background contributions. In particular a hard cut on the leading photon, $p_{T}^{\gamma}>m_{\gamma \gamma} / 2$ and an invariant mass cut on the diphoton pair, $m_{\gamma \gamma} \in[123.5,126.5] \mathrm{GeV}$, are applied. Moreover, high $S_{T}$ (defined as the scalar sum of the missing transverse momentum and the $p_{T}$ of the reconstructed leptons and jets) is required to fully exploit the large activity arising from the decay of pair produced heavy quarks. The events are further categorised in fully hadronic and leptonic channels, where at least one lepton, generally arising from a top quark decay, is required.

We thus propose to adopt a similar strategy to target the $S$ resonance arising from a heavy quark decay in the leptonic channel. We adopt in particular the following event selection criteria

- 2 photons with $p_{T}>10 \mathrm{GeV}$ and within the kinematic acceptance of the detector,

- $m_{\gamma \gamma} \in m_{S} \pm 5 \%$, 
- Leading photon with $p_{T}>m_{\gamma \gamma} / 2$,

- Second leading photon with $p_{T}>25 \mathrm{GeV}$,

- At least one lepton with $p_{T}>20 \mathrm{GeV}$,

- $S_{T}=E_{T}^{\mathrm{miss}}+H_{T}+\sum_{i=1}^{n_{l e p}} p_{T}^{i}>770 \mathrm{GeV}$,

where $H_{T}$ is the scalar sum of the transverse momentum of the reconstructed jets in the event.

For the $8 \mathrm{TeV}$ analysis the main contribution to the background in the leptonic channel comes from the non resonant processes with a yield of 0.11 events with $19.7 \mathrm{fb}^{-1}$ of integrated luminosity. This background is however estimated from data driven techniques, which are difficult to be accurately reproduced in a Monte Carlo simulation. Moreover, the decomposition into the $t \bar{t} \gamma \gamma, t \gamma \gamma$ and $\gamma \gamma+$ jets component is not specified in [37]. In order to estimate the non resonant background for the events selection proposed above we thus adopt the following strategy. We rescale each component of the non resonant background ( $\gamma \gamma t \bar{t}, \gamma \gamma t$ and $\gamma \gamma+$ jets) by taking into account both the increase in cross section due to the rescaling of the parton luminosity when going from 8 to $13 \mathrm{TeV}$ of centre of mass energy as well as the difference in acceptance (at the parton level) for reconstructing a $\gamma \gamma$ invariant mass of $750 \mathrm{GeV}$, with respect to $125 \mathrm{GeV}$, with a leading photon with $p_{T}>m_{\gamma \gamma} / 2$. We then obtain three rescaling factors, one for each non resonant background contribution, and we conservatively rescale the $8 \mathrm{TeV}$ background with the highest of these factors, $\sim 0.36$. With this strategy we obtain an event yield of 0.04 events with $19.7 \mathrm{fb}^{-1}$ at the $13 \mathrm{TeV}$ LHC, while the resonant contribution, that could arise from a the $t \bar{t} S$ associated production, can be safely neglected due to the small $S t \bar{t}$ coupling that follows from the small mixing assumption. With such a small number of background events, a more refined estimate is clearly not necessary. Event yields for different integrated luminosity assumptions are then easily obtained as $0.04 \times \mathcal{L} /\left(19.7 \mathrm{fb}^{-1}\right)$.

To calculate the signal cross sections for both the $g g \rightarrow S \rightarrow \gamma \gamma$ and $p p \rightarrow T \bar{T}$ processes, we implement the Lagrangian of eq. (2.1) in the UFO [50] format through the Feynrules [51] package and use MadGraph5_aMC@NLO [52] as event generator. Parton showering, hadronisation and decay of unstable particles have been performed through PYTHIA v6.4 [53] while Delphes v3.2.0 [54] has been employed for a fast detector simulation. Jets have been reconstructed with FastJet [55], via the anti- $k_{T}$ [56] algorithm with cone radius 0.5 , using a tuned CMS detector card suitable for performing an analysis with MadAnalysis5 [57].

We have then generated signal samples for the processes

$$
\begin{aligned}
& g g \rightarrow S \rightarrow \gamma \gamma \\
& p p \rightarrow T \bar{T} \rightarrow S t+X \text { (+h.c.), } S \rightarrow \gamma \gamma
\end{aligned}
$$

scanning over the following parameters values

- $y_{S}=2,4$ 

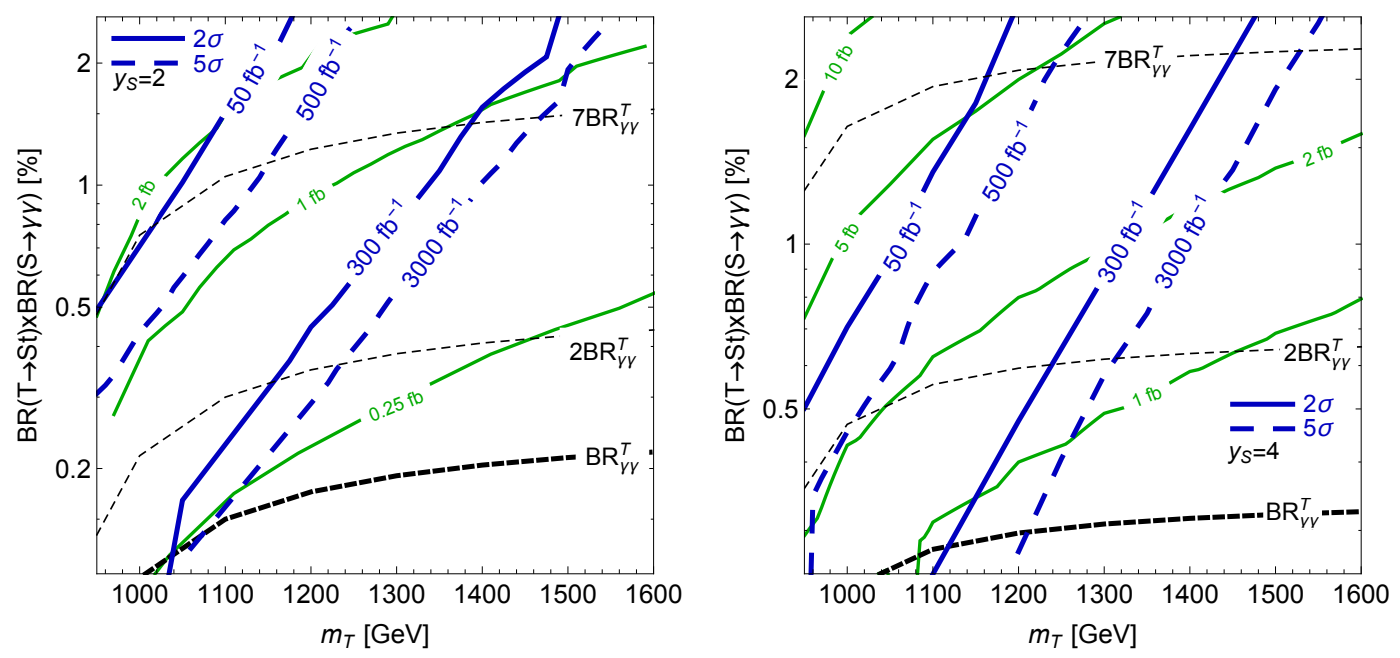

Figure 2. $2 \sigma$ and $5 \sigma$ sensitivity at the $13 \mathrm{TeV}$ LHC for the $p p \rightarrow \bar{T} T \rightarrow 2 \gamma+\ell+X$ search with various integrated luminosity options (see figure) in the $m_{T}-\mathrm{BR}(T \rightarrow S t) \mathrm{BR}(S \rightarrow \gamma \gamma)$ plane. The black dashed lines represent various branching ratio predictions for the model, with $\mathrm{BR}_{\gamma \gamma}^{T}$ the prediction with just one extra quark $T$. The green line are isocontours of constant $\sigma(g g \rightarrow \gamma \gamma)$. $y_{S}=2(4)$ is assumed in the left (right) panel.

- $M \in[1000-1500] \mathrm{GeV}$

- $\operatorname{BR}(S \rightarrow \gamma \gamma) \in\left[\mathrm{BR}_{\gamma \gamma}^{T}, 7 \times \mathrm{BR}_{\gamma \gamma}^{T}\right]$

where $\mathrm{BR}_{\gamma \gamma}^{T}$ is the $S \rightarrow \gamma \gamma$ branching ratio including only the $T$ quark. Heavy quark pair production cross sections have been normalised to the NNLO prediction computed with HATHOR [58], while we have applied a $k$-factor of $\sim 1.1$ to the $\gamma \gamma$ production cross section through gluon fusion as computed by MadGraph5_aMC@NLO. For this choices of parameters the LHC $8 \mathrm{TeV}$ limits on resonance searches from $j j$ [59, 60], $Z Z$ [61], $Z \gamma$ [62] and $\gamma \gamma[63,64]$ are fulfilled. Moreover, preliminary $13 \mathrm{TeV}$ results in the $Z Z$ final state [65] as well as limits from ATLAS searches for light dijet resonances are satisfied [66].

Our results for the $13 \mathrm{TeV}$ reach of the LHC for VLQ are shown in figure 2 in the $m_{T^{-}}$ $\operatorname{BR}(T \rightarrow S t) \times \operatorname{BR}(S \rightarrow \gamma \gamma)$ plane. The blue lines represent the $2 \sigma$ (solid) and $5 \sigma$ (dashed) isocontours, with the significance evaluated as $\alpha=\mathrm{N}_{S} / \sqrt{\mathrm{N}_{S}+\mathrm{N}_{B}}$, while the green lines are isocontours of constant $\sigma(g g \rightarrow \gamma \gamma)$.

In the right panel of figure 2 we see that for $y_{S}=4$, and assuming $\operatorname{BR}(S \rightarrow \gamma \gamma)=\mathrm{BR}_{\gamma \gamma}^{T}$, up to $m_{T} \sim 1150 \mathrm{GeV}$ can be probed at the $2 \sigma$ level via the $T \rightarrow S t$ decay channel with $\mathcal{L}=300 \mathrm{fb}^{-1}$. Allowing extra particles to increase $\operatorname{BR}(S \rightarrow \gamma \gamma)$ will give the possibility to probe higher values of $m_{T}$, up to $m_{T} \sim 1400 \mathrm{GeV}$ with $\operatorname{BR}(S \rightarrow \gamma \gamma)=4 \times \mathrm{BR}_{\gamma \gamma}^{T}$. Exploiting this channel for discovery will roughly require a factor 6 increase in luminosity. Note that the non observation from ATLAS and CMS of a signal excess in the diphoton mass spectrum with $\sim \mathcal{L}=13 \mathrm{fb}^{-1}$ sets a limit of $\sim 2 \mathrm{fb}$ on $\sigma(g g \rightarrow \gamma \gamma)[67,68]$.

Similar considerations hold for smaller values of $y_{s}$, for example $y_{S}=2$ (left panel of figure 2), where the constraints arising from diphoton resonant search are now less stringent and where, for $\operatorname{BR}(S \rightarrow \gamma \gamma)=\mathrm{BR}_{\gamma \gamma}^{T}$, the proposed search strategy attain a weaker limit. 
Our analysis can be clearly adapted for different values of the $S$ mass with respect to the one analysed. In the case of a higher $S$ mass the SM background will be further reduced with respect to the one previously estimated, mainly due to the tighter cut on the invariant mass of the $\gamma \gamma$ system while this selection will marginally impact the signal acceptance, since the two photon will still reconstruct the $S$ invariant mass. We thus expect an overall better sensitivity for higher $S$ masses, which will however probe a higher minimum $m_{T}$ mass, $m_{T}>m_{S}+m_{t}$, and with a smaller $g g \rightarrow \gamma \gamma$ rate. Conversely, a smaller $S$ mass will cause a reduction of our estimated LHC reach and a higher $g g \rightarrow \gamma \gamma$ rate. In the case of a narrow $S$, this loss in sensitivity could be partially compensated by a tightening of the $\gamma \gamma$ invariant mass cut below the assumed value of $5 \%$.

We conclude this section by stressing that the plane chosen for displaying our results is well suited for a general reinterpretation. In fact, $m_{T}$ uniquely sets the pair production rate of the heavy quark while the combination $\operatorname{BR}(T \rightarrow S t) \times \operatorname{BR}(S \rightarrow \gamma \gamma)$ determines the physical cross section (before selection acceptances and decays of SM particles) in the search channel considered. The projected limits obtained in this analysis can then be applied to any model featuring a heavy quark decaying into a scalar resonance $S$ and a top quark.

\section{Impact on other VLQ searches}

One important question that ought to be addressed is whether and how the high $T \rightarrow S t$ rate that can be obtained in this simplified scenario will affect the standard VLQs search strategies, tailored for the direct decay of $T$ into SM final states. These searches are mainly designed to be sensitive to the processes

$$
\begin{aligned}
& p p \rightarrow T \bar{T} \rightarrow W^{+} W^{-} b \bar{b} \\
& p p \rightarrow T \bar{T} \rightarrow Z t Z \bar{t} \rightarrow W^{+} W^{-} b \bar{b} Z Z \\
& p p \rightarrow T \bar{T} \rightarrow H t H \bar{t} \rightarrow W^{+} W^{-} b \bar{b} H H
\end{aligned}
$$

in various final states [37], including fully hadronic channels. While in section 3 we have exploited the similarity of the $H t H \bar{t}$ with the $S t S \bar{t}$ channel in the $2 \gamma+\ell+X$ final states to design an analysis sensitive to the $T \rightarrow S t$ decay mode, ${ }^{4}$ we now analyse the similarities with the other two decay patterns.

Pair produced VLQs decaying into the heavy $S$ resonance will undergo a $T \bar{T} \rightarrow S t S \bar{t} \rightarrow$ $S S W^{+} W^{-} b \bar{b}$ decay chain. Since in our scenario the $S$ is expected to decay almost $100 \%$ of the times into a pair of gluons one finally obtains a $W^{+} W^{-} b \bar{b}+4 j$ final state. The similarity with the final states arising from $T \rightarrow W^{+} b$ and $T \rightarrow Z t$ is clearly manifest. It is thus natural to expect that standard VLQ searches could be sensitive to the $T \rightarrow S t$ decay without any significant modifications of the analysis strategies and selection cuts. ${ }^{5}$ We illustrate this for the case of an analysis targeting the $Z t Z t$ final state exploited by the CMS collaboration in performing VLQ searches during LHC Run-1 [37]. This search

\footnotetext{
${ }^{4}$ Note that in case of a heavy quark with charge $-1 / 3$ decaying into $S b$ similar search strategies can be proposed.

${ }^{5}$ See however ref. [69] for a proposal to probe the $W^{+} W^{-} b \bar{b}+4 j$ final state with multi-jet searches designed for supersymmetric scenarios.
} 


\begin{tabular}{|c|c|c|c|c|c|}
\hline $\mathcal{L}\left[\mathrm{fb}^{-1}\right]$ & $\mathrm{N}_{\text {bkg. }}$ & $\mathrm{N}_{\mathrm{BP}_{1}}$ & $\alpha_{\mathrm{BP}_{1}}$ & $\mathrm{~N}_{\mathrm{BP}_{2}}$ & $\alpha_{\mathrm{BP}_{2}}$ \\
\hline 100 & 439 & 26 & $1.2 \sigma$ & 22 & $1.0 \sigma$ \\
300 & 1317 & 79 & $2.1 \sigma$ & 67 & $1.8 \sigma$ \\
1000 & 4390 & 262 & $3.8 \sigma$ & 223 & $3.3 \sigma$ \\
3000 & 13170 & 786 & $6.7 \sigma$ & 674 & $5.8 \sigma$ \\
\hline
\end{tabular}

Table 1. Number of background and signal events as well as the statistical significance $\alpha$ for two benchmark points with $m_{T}=1000 \mathrm{GeV}, \mathrm{BR}(S \rightarrow \gamma \gamma)=2 \times \mathrm{BR}_{\gamma \gamma}^{T}\left(\mathrm{BP}_{1}\right)$ and $m_{T}=1100 \mathrm{GeV}$, $\mathrm{BR}(S \rightarrow \gamma \gamma)=\mathrm{BR}_{\gamma \gamma}^{T}\left(\mathrm{BP}_{2}\right)$ after the $O S 2$ selection with $H_{T}>1000 \mathrm{GeV}$ and $S_{T}>1500 \mathrm{GeV}$ for various integrated luminosity options.
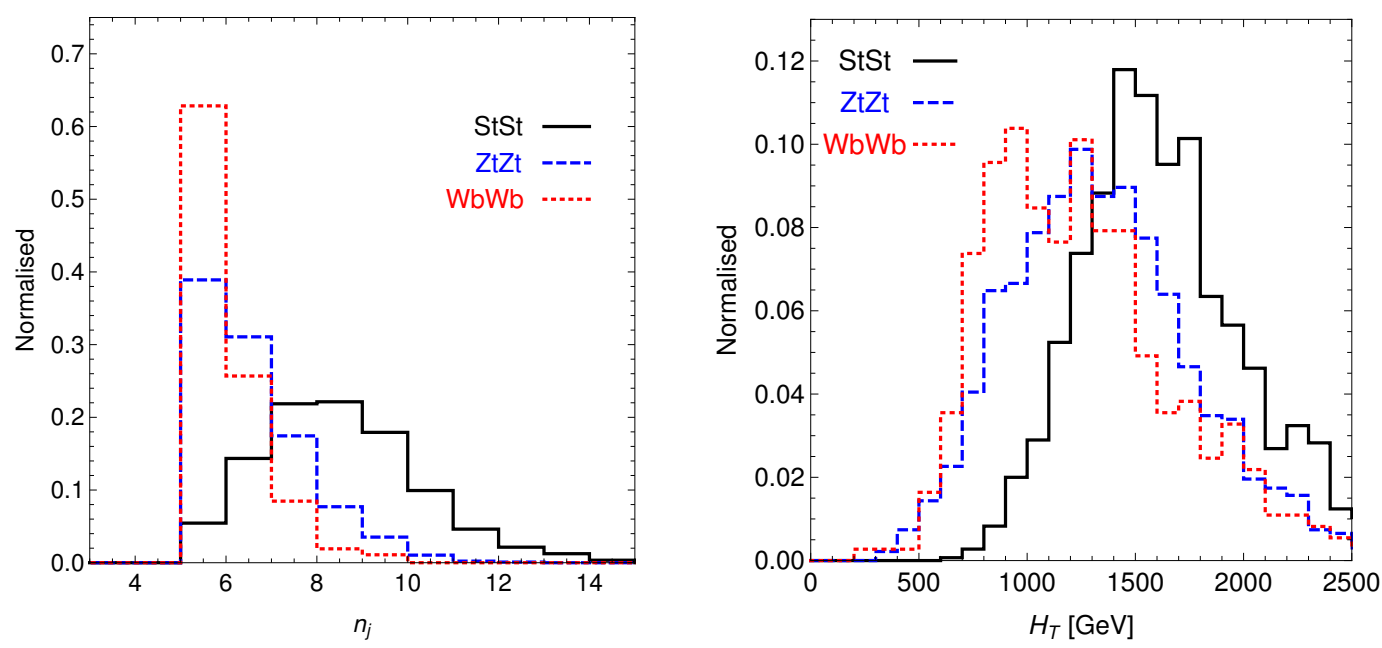

Figure 3. Jet multiplicity and $H_{T}$ distributions for the case of a pair of heavy quarks with mass $1000 \mathrm{GeV}$ decaying into the $W^{+} b W^{-} \bar{b}, Z t Z \bar{t}$ and $S t S \bar{t}$ final states. Distributions are normalised to unity.

channel, named $O S 2$, requires exactly two opposite sign leptons (electrons or muons) and at least five jets, two of which must be identified as $b$ jets, and required to have $\Delta \mathrm{R}>0.3$ from the selected leptons. The invariant mass of the dilepton pair is demanded to be greater than $20 \mathrm{GeV}$ and $E_{T}^{\text {miss }}>30 \mathrm{GeV}$ is applied. Finally cuts on $H_{T}>500 \mathrm{GeV}$ and $S_{T}>1000 \mathrm{GeV}$ are imposed. The main background for this channel is $t \bar{t}+n j$. For two benchmark points with $y_{S}=8,{ }^{6}$ we check the sensitivity of the $13 \mathrm{TeV}$ LHC to the $W^{+} W^{-} b \bar{b}+4 j$ final state for a selection similar to CMS' $O S 2$. We however impose tighter cuts on $H_{T}>1200 \mathrm{GeV}$ and $S_{T}>1700 \mathrm{GeV}$ in order to take advantage of the fact that jets arising from the $S$ decay will in general be harder than the ones arising from the $Z$ $(W)$ in the $T \rightarrow Z t(W b)$ case. The number of background and signal events for the two benchmark points, corresponding to $m_{T}=1000 \mathrm{GeV}, \mathrm{BR}(S \rightarrow \gamma \gamma)=2 \times \mathrm{BR}_{\gamma \gamma}^{T}\left(\mathrm{BP}_{1}\right)$ and $m_{T}=1100 \mathrm{GeV}, \mathrm{BR}(S \rightarrow \gamma \gamma)=\mathrm{BR}_{\gamma \gamma}^{T}\left(\mathrm{BP}_{2}\right)$ are reported in table 1 for various choices of the integrated luminosity together with the value of the statistical significance $\alpha$.

\footnotetext{
${ }^{6}$ Note that the chosen representative value $y_{S}=8$ is actually excluded by the upper bound on the diphoton cross-section. We expect however, qualitatively similar results for allowed points with $y_{S}=2,4$, with only a slight decrease in sensitivity due to the lower $\operatorname{BR}(T \rightarrow S t)$, see figure 1 .
} 
Finally, in the fortunate event that a signal is observed e.g., in a channel designed for the $Z t Z t$ final state (as the $O S 2$ previously described) it is interesting to note that it might be possible to decipher whether this arises from the decay $T \rightarrow S t$ or $T \rightarrow Z t$. This can be achieved by exploiting the larger jet multiplicity and the higher hadronic activity found in the $S t S \bar{t}$ final state as compared to $W^{+} b W^{-} \bar{b}, Z t Z \bar{t}$, see the corresponding distributions in figure 3.

\section{Conclusions}

The decay of a heavy vector-like quark into a scalar and a light quark provide a useful search channel for VLQ at the LHC, in particular when additional particles further boost the branching ratio of the scalar into diphotons. Using a $\mathrm{SU}(2)_{L}$ singlet heavy quark and a $750 \mathrm{GeV}$ scalar as an illustration, we have shown that the $13 \mathrm{TeV}$ LHC can probe VLQ decays dominantly into the top quark and the $S$ resonance in the $2 \gamma+\ell+X$ channels for a large region of the parameter space compatible with upper limits on the $p p \rightarrow S \rightarrow \gamma \gamma$ cross section. The projected reach we have obtained for this channel can be directly applied to other choices of representations for VLQs since we have expressed it in terms of the heavy quark mass and the combination $\operatorname{BR}(T \rightarrow S t) \times \operatorname{BR}(S \rightarrow \gamma \gamma)$ that uniquely fixes both the $T$ pair production cross section and the event rate before kinematic selections and decay of SM particles. In addition, the decay of the VLQ into a scalar resonance can also lead to a $W^{+} W^{-} b \bar{b}+4 j$ final state. We have shown that current VLQ searches tailored for the direct decay of the heavy quark into SM states can be sensitive to this final state without a drastic modification of the selection cuts. Although we have focused our analysis on the specific case of a $750 \mathrm{GeV}$ scalar, our results can easily be extended to any high mass state lighter than the vector-like quark and decaying into diphotons. Finally, in the event an excess is observed in an analysis targeting pair produced heavy quarks we observe that various kinematic distributions can be exploited to disentangle whether this excess arises from the $T \rightarrow S t$ decay or from standard heavy quark decay modes.

\section{Acknowledgments}

We wish to thank Fawzi Boudjema, Devdatta Majumder and Emanuele Re for useful discussions. This work is supported by the "Investissements d'avenir, Labex ENIGMASS", by the French ANR, Project DMAstro-LHC, ANR-12-BS05-006, by the Research Executive Agency (REA) of the European Union under the Grant Agreement PITN-GA-2012-316704 ("HiggsTools") and by the Indo French LIA THEP (Theoretical High Energy Physics) of the CNRS.

Open Access. This article is distributed under the terms of the Creative Commons Attribution License (CC-BY 4.0), which permits any use, distribution and reproduction in any medium, provided the original author(s) and source are credited. 


\section{References}

[1] P.W. Graham, A. Ismail, S. Rajendran and P. Saraswat, A little solution to the little hierarchy problem: a vector-like generation, Phys. Rev. D 81 (2010) 055016 [arXiv:0910.3020] [INSPIRE].

[2] S.P. Martin, Extra vector-like matter and the lightest Higgs scalar boson mass in low-energy supersymmetry, Phys. Rev. D 81 (2010) 035004 [arXiv:0910.2732] [INSPIRE].

[3] R. Dermisek, Unification of gauge couplings in the standard model with extra vectorlike families, Phys. Rev. D 87 (2013) 055008 [arXiv: 1212.3035] [INSPIRE].

[4] M. Buchkremer and A. Schmidt, Long-lived heavy quarks: a review, Adv. High Energy Phys. 2013 (2013) 690254 [arXiv: 1210.6369] [INSPIRE].

[5] M. Buchkremer, G. Cacciapaglia, A. Deandrea and L. Panizzi, Model independent framework for searches of top partners, Nucl. Phys. B 876 (2013) 376 [arXiv:1305.4172] [INSPIRE].

[6] S. Gopalakrishna, T. Mandal, S. Mitra and G. Moreau, LHC signatures of warped-space vectorlike quarks, JHEP 08 (2014) 079 [arXiv:1306.2656] [INSPIRE].

[7] S.A.R. Ellis, R.M. Godbole, S. Gopalakrishna and J.D. Wells, Survey of vector-like fermion extensions of the Standard Model and their phenomenological implications, JHEP 09 (2014) 130 [arXiv: 1404.4398] [INSPIRE].

[8] G. Cacciapaglia and A. Parolini, Light 't Hooft top partners, Phys. Rev. D 93 (2016) 071701 [arXiv: 1511.05163] [INSPIRE].

[9] M. Low, A. Tesi and L.-T. Wang, A pseudoscalar decaying to photon pairs in the early LHC Run 2 data, JHEP 03 (2016) 108 [arXiv: 1512.05328] [INSPIRE].

[10] R. Franceschini et al., What is the $\gamma \gamma$ resonance at 750 GeV?, JHEP 03 (2016) 144 [arXiv: 1512.04933] [INSPIRE].

[11] B. Batell, S. Jung and H.M. Lee, Singlet assisted vacuum stability and the Higgs to diphoton rate, JHEP 01 (2013) 135 [arXiv: 1211.2449] [INSPIRE].

[12] M.-L. Xiao and J.-H. Yu, Stabilizing electroweak vacuum in a vectorlike fermion model, Phys. Rev. D 90 (2014) 014007 [arXiv:1404.0681] [INSPIRE].

[13] ATLAS collaboration, Search for resonances decaying to photon pairs in $3.2 \mathrm{fb}^{-1}$ of $\mathrm{pp}$ collisions at $\sqrt{s}=13$ TeV with the ATLAS detector, ATLAS-CONF-2015-081 (2015).

[14] CMS collaboration, Search for new physics in high mass diphoton events in proton-proton collisions at $\sqrt{s}=13$ TeV, CMS-PAS-EXO-15-004 (2015).

[15] ATLAS collaboration, Search for resonances in diphoton events with the ATLAS detector at $\sqrt{s}=13$ TeV, ATLAS-CONF-2016-018 (2016).

[16] ATLAS collaboration, Search for resonances in diphoton events at $\sqrt{s}=13$ TeV with the ATLAS detector, JHEP 09 (2016) 001 [arXiv: 1606.03833] [INSPIRE].

[17] CMS Collaboration, Search for new physics in high mass diphoton events in $3.3 \mathrm{fb}^{-1}$ of proton-proton collisions at $\sqrt{s}=13$ TeV and combined interpretation of searches at 8 Te $V$ and 13 TeV, CMS-PAS-EXO-16-018 (2016).

[18] CMS collaboration, Search for resonant production of high-mass photon pairs in proton-proton collisions at $\sqrt{s}=8$ and 13 TeV, Phys. Rev. Lett. 117 (2016) 051802 [arXiv: 1606.04093] [INSPIRE]. 
[19] K. Harigaya and Y. Nomura, Composite models for the $750 \mathrm{GeV}$ diphoton excess, Phys. Lett. B 754 (2016) 151 [arXiv: 1512.04850] [INSPIRE].

[20] Y. Mambrini, G. Arcadi and A. Djouadi, The LHC diphoton resonance and dark matter, Phys. Lett. B 755 (2016) 426 [arXiv: 1512.04913] [INSPIRE].

[21] M. Backovic, A. Mariotti and D. Redigolo, Di-photon excess illuminates Dark Matter, JHEP 03 (2016) 157 [arXiv: 1512.04917] [INSPIRE].

[22] A. Angelescu, A. Djouadi and G. Moreau, Scenarii for interpretations of the LHC diphoton excess: two Higgs doublets and vector-like quarks and leptons, Phys. Lett. B 756 (2016) 126 [arXiv: 1512.04921] [INSPIRE].

[23] Y. Nakai, R. Sato and K. Tobioka, Footprints of new strong dynamics via anomaly and the $750 \mathrm{GeV}$ diphoton, Phys. Rev. Lett. 116 (2016) 151802 [arXiv:1512.04924] [INSPIRE].

[24] S. Knapen, T. Melia, M. Papucci and K. Zurek, Rays of light from the LHC, Phys. Rev. D 93 (2016) 075020 [arXiv: 1512.04928] [INSPIRE].

[25] D. Buttazzo, A. Greljo and D. Marzocca, Knocking on new physics's door with a scalar resonance, Eur. Phys. J. C 76 (2016) 116 [arXiv:1512.04929] [INSPIRE].

[26] S. Di Chiara, L. Marzola and M. Raidal, First interpretation of the $750 \mathrm{GeV}$ diphoton resonance at the LHC, Phys. Rev. D 93 (2016) 095018 [arXiv:1512.04939] [INSPIRE].

[27] R.S. Gupta, S. Jäger, Y. Kats, G. Perez and E. Stamou, Interpreting a $750 \mathrm{GeV}$ Diphoton Resonance, JHEP 07 (2016) 145 [arXiv: 1512.05332] [INSPIRE].

[28] J. Gao, H. Zhang and H.X. Zhu, Diphoton excess at 750 GeV: gluon-gluon fusion or quark-antiquark annihilation?, Eur. Phys. J. C 76 (2016) 348 [arXiv:1512.08478] [INSPIRE].

[29] W. Altmannshofer et al., 750 GeV diphoton excess, Phys. Rev. D 93 (2016) 095015 [arXiv: 1512.07616] [INSPIRE].

[30] Y. Kats and M.J. Strassler, Resonances from QCD bound states and the $750 \mathrm{GeV}$ diphoton excess, JHEP 05 (2016) 092 [Erratum ibid. 07 (2016) 044] [arXiv: 1602.08819] [INSPIRE].

[31] A. Strumia, Interpreting the $750 \mathrm{GeV}$ digamma excess: a review, arXiv:1605.09401 [INSPIRE].

[32] R. Franceschini et al., Digamma, what next?, JHEP 07 (2016) 150 [arXiv:1604.06446] [INSPIRE].

[33] F. Goertz, J.F. Kamenik, A. Katz and M. Nardecchia, Indirect constraints on the scalar di-photon resonance at the LHC, JHEP 05 (2016) 187 [arXiv: 1512.08500] [INSPIRE].

[34] A. Salvio, F. Staub, A. Strumia and A. Urbano, On the maximal diphoton width, JHEP 03 (2016) 214 [arXiv: 1602.01460] [INSPIRE].

[35] ATLAS collaboration, Search for scalar diphoton resonances with $15.4 \mathrm{fb}^{-1}$ of data collected at $\sqrt{s}=13 \mathrm{TeV}$ in 2015 and 2016 with the ATLAS detector, ATLAS-CONF-2016-059 (2016).

[36] CMS Collaboration, Search for resonant production of high mass photon pairs using $12.9 \mathrm{fb}^{-1}$ of proton-proton collisions at $\sqrt{s}=13 \mathrm{TeV}$ and combined interpretation of searches at 8 and $13 \mathrm{TeV}$, CMS-PAS-EXO-16-027 (2016).

[37] CMS collaboration, Search for vector-like charge $2 / 3$ T quarks in proton-proton collisions at $\sqrt{s}=8$ TeV, Phys. Rev. D 93 (2016) 012003 [arXiv:1509.04177] [InSPIRE]. 
[38] CMS collaboration, Search for pair-produced vectorlike B quarks in proton-proton collisions at $\sqrt{s}=8$ TeV, Phys. Rev. D 93 (2016) 112009 [arXiv:1507.07129] [INSPIRE].

[39] ATLAS collaboration, Search for production of vector-like quark pairs and of four top quarks in the lepton-plus-jets final state in pp collisions at $\sqrt{s}=8$ TeV with the ATLAS detector, JHEP 08 (2015) 105 [arXiv: 1505.04306] [INSPIRE].

[40] ATLAS collaboration, Search for pair production of a new heavy quark that decays into a $W$ boson and a light quark in pp collisions at $\sqrt{s}=8$ TeV with the ATLAS detector, Phys. Rev. D 92 (2015) 112007 [arXiv: 1509.04261] [INSPIRE].

[41] D. Barducci et al., Framework for model independent analyses of multiple extra quark scenarios, JHEP 12 (2014) 080 [arXiv: 1405.0737] [INSPIRE].

[42] ATLAS collaboration, Search for production of vector-like top quark pairs and of four top quarks in the lepton-plus-jets final state in pp collisions at $\sqrt{s}=13$ TeV with the ATLAS detector, ATLAS-CONF-2016-013 (2016).

[43] J. Kearney, A. Pierce and J. Thaler, Top partner probes of extended Higgs sectors, JHEP 08 (2013) 130 [arXiv:1304.4233] [InSPIRE].

[44] E. Coluccio Leskow, T.A.W. Martin and A. de la Puente, Vector-like quarks with a scalar triplet, Phys. Lett. B 743 (2015) 366 [arXiv:1409.3579] [InSPIRE].

[45] A. Anandakrishnan, J.H. Collins, M. Farina, E. Kuflik and M. Perelstein, Odd top partners at the LHC, Phys. Rev. D 93 (2016) 075009 [arXiv:1506.05130] [InSPIRE].

[46] J. Serra, Beyond the minimal top partner decay, JHEP 09 (2015) 176 [arXiv:1506.05110] [INSPIRE].

[47] K. Das and S.K. Rai, 750 GeV diphoton excess in a U(1) hidden symmetry model, Phys. Rev. D 93 (2016) 095007 [arXiv: 1512.07789] [InSPIRE].

[48] J.H. Collins, C. Csáki, J.A. Dror and S. Lombardo, Novel kinematics from a custodially protected diphoton resonance, Phys. Rev. D 93 (2016) 115001 [arXiv:1603.09350] [INSPIRE].

[49] A. Djouadi, The Anatomy of electro-weak symmetry breaking. I: the Higgs boson in the standard model, Phys. Rept. 457 (2008) 1 [hep-ph/0503172] [INSPIRE].

[50] C. Degrande, C. Duhr, B. Fuks, D. Grellscheid, O. Mattelaer and T. Reiter, UFO - The Universal FeynRules Output, Comput. Phys. Commun. 183 (2012) 1201 [arXiv:1108.2040] [INSPIRE].

[51] A. Alloul, N.D. Christensen, C. Degrande, C. Duhr and B. Fuks, FeynRules 2.0 - A complete toolbox for tree-level phenomenology, Comput. Phys. Commun. 185 (2014) 2250 [arXiv: 1310.1921] [INSPIRE].

[52] J. Alwall et al., The automated computation of tree-level and next-to-leading order differential cross sections and their matching to parton shower simulations, JHEP 07 (2014) 079 [arXiv: 1405.0301] [INSPIRE].

[53] T. Sjöstrand, S. Mrenna and P.Z. Skands, PYTHIA 6.4 physics and manual, JHEP 05 (2006) 026 [hep-ph/0603175] [INSPIRE].

[54] DELPHES 3 collaboration, J. de Favereau et al., DELPHES 3, a modular framework for fast simulation of a generic collider experiment, JHEP 02 (2014) 057 [arXiv:1307.6346] [INSPIRE]. 
[55] M. Cacciari, G.P. Salam and G. Soyez, FastJet user manual, Eur. Phys. J. C 72 (2012) 1896 [arXiv: 1111.6097] [INSPIRE].

[56] M. Cacciari, G.P. Salam and G. Soyez, The anti- $k_{t}$ jet clustering algorithm, JHEP 04 (2008) 063 [arXiv: 0802.1189] [INSPIRE].

[57] E. Conte, B. Fuks and G. Serret, MadAnalysis 5, a user-friendly framework for collider phenomenology, Comput. Phys. Commun. 184 (2013) 222 [arXiv:1206.1599] [INSPIRE].

[58] M. Aliev et al., HATHOR: HAdronic Top and Heavy quarks crOss section calculatoR, Comput. Phys. Commun. 182 (2011) 1034 [arXiv:1007.1327] [InSPIRE].

[59] ATLAS collaboration, Search for new phenomena in the dijet mass distribution using pp collision data at $\sqrt{s}=8 \mathrm{TeV}$ with the ATLAS detector, Phys. Rev. D 91 (2015) 052007 [arXiv: 1407.1376] [INSPIRE].

[60] CMS collaboration, Search for resonances decaying to dijet final states at $\sqrt{s}=8 \mathrm{TeV}$ with scouting data, CMS-PAS-EXO-14-005 (2014).

[61] ATLAS collaboration, Search for an additional, heavy Higgs boson in the $H \rightarrow Z Z$ decay channel at $\sqrt{s}=8 \mathrm{TeV}$ in pp collision data with the ATLAS detector, Eur. Phys. J. C 76 (2016) 45 [arXiv: 1507.05930].

[62] ATLAS collaboration, Search for new resonances in $W \gamma$ and $Z \gamma$ final states in pp collisions at $\sqrt{s}=8 \mathrm{TeV}$ with the ATLAS detector, Phys. Lett. B 738 (2014) 428 [arXiv:1407.8150] [INSPIRE].

[63] CMS collaboration, Search for new resonances in the diphoton final state in the range between 150 and $850 \mathrm{GeV}$ in pp collisions at $\sqrt{s}=T e V$, CMS-PAS-HIG-14-006 (2014).

[64] ATLAS collaboration, Search for high-mass diphoton resonances in pp collisions at $\sqrt{s}=8$ TeV with the ATLAS detector, Phys. Rev. D 92 (2015) 032004 [arXiv:1504.05511] [INSPIRE].

[65] ATLAS collaboration, Search for diboson resonances in the llqq final state in pp collisions at $\sqrt{s}=13 \mathrm{TeV}$ with the ATLAS detector, ATLAS-CONF-2015-071 (2015).

[66] ATLAS collaboration, Search for light dijet resonances with the ATLAS detector using a trigger-level Analysis in LHC pp collisions at $\sqrt{s}=13 \mathrm{TeV}$, ATLAS-CONF-2016-030 (2016).

[67] CMS collaboration, Search for high-mass diphoton resonances in proton-proton collisions at $13 \mathrm{TeV}$ and combination with $8 \mathrm{TeV}$ search, arXiv:1609.02507 [INSPIRE].

[68] ATLAS collaboration, Search for scalar diphoton resonances with $15.4 \mathrm{fb}^{-1}$ of data collected at $\sqrt{s}=13 \mathrm{TeV}$ in 2015 and 2016 with the ATLAS detector, ATLAS-CONF-2016-059 (2016).

[69] P. Agrawal, J. Fan, B. Heidenreich, M. Reece and M. Strassler, Experimental considerations motivated by the diphoton excess at the LHC, JHEP 06 (2016) 082 [arXiv: 1512.05775] [INSPIRE]. 